\title{
The Operator-valued Poisson Kernel and its Applications
}

\author{
ISABELLE CHALENDAR
}

\section{INTRODUCTION}

Let $\mathcal{H}$ be a complex Hilbert space and let $\mathcal{L}(\mathcal{H})$ denote the algebra of all linear and bounded mappings from $\mathcal{H}$ to $\mathcal{H}$. For $T \in \mathcal{L}(\mathcal{H})$, its spectrum $\sigma(T)$ is the nonempty compact subset of $\mathbb{C}$ consisting of all $\lambda \in \mathbb{C}$ such that $T-\lambda I d$ is non-invertible in $\mathcal{L}(\mathcal{H})$. Its point spectrum $\sigma_{p}(T)$ is the (possibly empty) subset of $\sigma(T)$ consisting of those $\lambda \in \mathbb{C}$ such that $\operatorname{ker}(T-\lambda I d) \neq\{0\}$. We write $\mathbb{D}$ for the open unit disc in $\mathbb{C}$, and $\mathbb{T}$ for the unit circle. The spaces $L^{p}=L^{p}(\mathbb{T})$, $1 \leq p \leq \infty$ are the usual Lebesgue function spaces relative to normalized Lebesgue measure on $\mathbb{T}$.

As usual, we define the Hardy space $H^{2}=H^{2}(\mathbb{D})$ as the space of all functions $f: z \mapsto \sum_{n=0}^{\infty} a_{n} z^{n}$ for which the norm $\|f\|=$ $\left(\sum_{n=0}^{\infty}\left|a_{n}\right|^{2}\right)^{1 / 2}$ is finite. It is well known that $H^{2}(\mathbb{D})$ may be regarded isometrically as a closed subspace $H^{2}(\mathbb{T})$ of $L^{2}$ [31, 25], by identifying the Taylor coefficients of $f$ with the Fourier coefficients of an $L^{2}(\mathbb{T})$ function. The Hardy space $H^{\infty}$ is the set of bounded and analytic functions on $\mathbb{D}$, which is isometrically isomorphic to $H^{\infty}(\mathbb{T}):=\left\{f \in L^{\infty}(\mathbb{T}): \hat{f}(n)=0, n<0\right\}$ via the mapping that takes a function into its radial limit. The space $H_{0}^{1}$ will denote the set of functions $f \in L^{1}$ such that $\hat{f}(n)=0, n \leq 0$, which can be naturally identified with the predual of $H^{\infty}(\mathbb{T}) . \mathcal{M}(\mathbb{T})$ is the set of all (finite) complex measures on $\mathbb{T}$.

The contents of this paper is mostly the contents of the talks that were given at the Belfast Functional Analysis Day 2002. The aim is to present in an as elementary as possible way some of the properties of the so-called operator-valued Poisson kernel and to describe in detail some of its applications in operator theory and harmonic analysis understandable by non-specialists. For further information see 
also $[13,14,18]$, where G. Cassier and T. Fack study the behaviour of contractions and operators similar to contractions, in the context of von Neumann algebras. We also mention the works of R. Curto and F. Vasilescu [47, 24, 48], where the authors use the operatorvalued Poisson kernel to define an $A\left(\mathbb{D}^{n}\right)$-functional calculus for an $n$-tuple of commuting operators $T=\left(T_{1}, \cdots, T_{n}\right)$ with $\sigma(T) \subset \mathbb{D}^{n}$. In [48], Vasilescu obtained a von Neumann's inequality and a series of dilation theory objects related to $T$ when $T_{1}^{*} T_{1}+\cdots T_{n}^{*} T_{n} \leq I d$.

The paper is organized as follows. In Section 2 we define and analyse the operator-valued Poisson kernel, especially its properties in common with the (scalar) Poisson kernel. The two main applications that are presented are a dilation free proof of the well-known von Neumann's inequality and the proof of the existence of a density of elementary spectral measure for contractions. Section 3 relies highly on Section 2 where we show how to calculate a functional calculus for absolutely continuous contraction, first for the disc algebra and then for $H^{\infty}$. The last sections are devoted to applications. In Section 4, after a short introduction concerning the invariant subspace problem, we will give an idea of the nature of S. Brown's approximation method, which gave birth to dual algebra theory. In particular, we present S. Brown's starting point, involving density of elementary spectral measure, and standard facts about the so-called class $\mathbb{A}$ and its subclasses $\mathbb{A}_{n, m}$. Finally, using the powerful tool of operator-valued Poisson kernel, we show how to prove that classes $\mathbb{A}_{n, m}$ are distinct. In Section 5, combining a result of J. Bourgain with a result concerning the expression of the density of elementary spectral measures associated with $b(T)$ (where $b$ is a finite Blaschke product and $T$ an absolutely continuous contraction) in terms of those associated with $T$, we obtain a factorization result for functions in $L^{1}$ by means of functions in $H^{2}(\mathbb{T})$.

In order to make this article as self-contained as possible, we will give the proofs of most of the results more specifically involving the operator-valued Poisson kernel. 


\section{The Operator-Valued Poisson Kernel}

For $r e^{i t} \in \mathbb{D}$, recall that the (scalar) Poisson kernel $P_{r, t}$ is defined by

$$
\begin{aligned}
P_{r, t}\left(e^{i \theta}\right) & =\frac{1}{1-r e^{i t} e^{-i \theta}}+\frac{1}{1-r e^{-i t} e^{i \theta}}-1 \\
& =\frac{1-r^{2}}{\left|1-r e^{i t} e^{-i \theta}\right|^{2}} \\
& =\sum_{n \geq 0} r^{n} e^{i n t} e^{-i n \theta}+\sum_{n \geq 0} r^{n} e^{-i n t} e^{i n \theta}-1 .
\end{aligned}
$$

Note that $P_{r, t}\left(e^{i \theta}\right) \geq 0$. Recall also that each function $f \in L^{1}$ has a natural harmonic extension into $\mathbb{D}$, which will also be denoted by $f$. The extension is defined by Poisson's formula:

$$
f\left(r e^{i t}\right)=\frac{1}{2 \pi} \int_{0}^{2 \pi} f\left(e^{i \theta}\right) P_{r, t}\left(e^{i \theta}\right) d \theta .
$$

In the sequel we will need the following theorem ([44], Chap. 11).

Theorem A. Let $\mathcal{E}$ be the set of harmonic functions $f$ on $\mathbb{D}$ such that

$$
\rho(f):=\sup _{0 \leq s<1} \int_{0}^{2 \pi}\left|f\left(s e^{i t}\right)\right| d t<\infty .
$$

For $\mu \in \mathcal{M}(\mathbb{T})$, denote by $P(\mu)$ the harmonic function on $\mathbb{D}$ defined by

$$
P(\mu)\left(r e^{i t}\right)=\frac{1}{2 \pi} \int_{0}^{2 \pi} P_{r, t}\left(e^{i \theta}\right) d \mu(\theta) .
$$

The mapping $\left\{\begin{aligned} \mathcal{M}(\mathbb{T}) & \longrightarrow \mathcal{E} \\ \mu & \longmapsto P(\mu)\end{aligned} \quad\right.$ is bijective with $\rho(P(\mu))=$ $\|\mu\|$. Moreover, $\lim _{r \rightarrow 1^{-}} P(\mu)\left(r e^{i t}\right)$ exists a.e. and is equal to the $L^{1}$-function called the Radon-Nikodym derivative of $\mu$, that is, the density of the absolutely continuous part of $\mu$.

Now, for $T \in \mathcal{L}(\mathcal{H})$ such that $\sigma(T) \subset \overline{\mathbb{D}}$ and for $r e^{i t} \in \mathbb{D}$, define the operator-valued Poisson kernel $K_{r, t}(T) \in \mathcal{L}(\mathcal{H})$ in the following way:

$$
K_{r, t}(T)=\left(I d-r e^{i t} T^{*}\right)^{-1}+\left(I d-r e^{-i t} T\right)^{-1}-I d .
$$


Remark 2.1. Note that

$$
\left(K_{r, t}(T)\right)^{*}=K_{r, t}(T) \text { and } K_{r,-t}(T)=K_{r, t}\left(T^{*}\right) .
$$

As for the (scalar) Poisson kernel, we have the following equalities:

Lemma 2.2. For $T \in \mathcal{L}(\mathcal{H})$ such that $\sigma(T) \subset \overline{\mathbb{D}}$, we have:

$$
\begin{aligned}
K_{r, t}(T) & =\left(I d-r e^{i t} T^{*}\right)^{-1}\left(I d-r^{2} T^{*} T\right)\left(I d-r e^{-i t} T\right)^{-1} \\
& =\left(I d-r e^{-i t} T\right)^{-1}\left(I d-r^{2} T T^{*}\right)\left(I d-r e^{i t} T^{*}\right)^{-1} \\
& =\sum_{n \geq 0} r^{n} e^{i n t} T^{* n}+\sum_{n \geq 0} r^{n} e^{-i n t} T^{n}-I d
\end{aligned}
$$

Proof: The two first equalities follow from the fact that

$$
\begin{aligned}
& \left(I d-r e^{i t} T^{*}\right) K_{r, t}(T)\left(I d-r e^{-i t} T\right)=I d-r^{2} T^{*} T \text { and } \\
& \left(I d-r e^{-i t} T\right) K_{r, t}(T)\left(I d-r e^{i t} T^{*}\right)=I d-r^{2} T T^{*} .
\end{aligned}
$$

Moreover, since $\sigma(T) \subset \overline{\mathbb{D}}$, we have $\lim _{n \rightarrow \infty}\left\|T^{n}\right\|^{1 / n} \leq 1$. For each $\varepsilon>0$, there exists $N \geq 1$ such that $\left\|T^{n}\right\| \leq(1+\varepsilon)^{n}$ for all $n \geq N$. Now, taking $r>0$ such that $r(1+\varepsilon)<1$, we get the convergence in norm of $\sum_{n \geq 0} r^{n} e^{i n t} T^{* n}+\sum_{n \geq 0} r^{n} e^{-i n t} T^{n}-I d$, which proves the last assertion of the lemma.

For $T \in \mathcal{L}(\mathcal{H})$ and $p \in \mathbb{C}[z]_{\mid \overline{\mathbb{D}}}$ a polynomial, it is natural to define $p(T) \in \mathcal{L}(\mathcal{H})$ in the following way:

$$
p(T)=\sum_{k=0}^{n} a_{k} T^{k} \quad \text { if } \quad p(z)=\sum_{k=0}^{n} a_{k} z^{k} .
$$

Actually, using the operator-valued Poisson kernel, there is another way to define $p(r T)$ for $0 \leq r<1$.

Lemma 2.3. Let $T \in \mathcal{L}(\mathcal{H})$ such that $\sigma(T) \subset \overline{\mathbb{D}}$. For all $r \in[0,1)$, we have:

$$
p(r T)=\frac{1}{2 \pi} \int_{0}^{2 \pi} p\left(e^{i t}\right) K_{r, t}(T) d t, p \in \mathbb{C}[z]_{\mid \overline{\mathbb{D}}} .
$$

Proof: $\quad$ Let $p(z)=\sum_{k=0}^{N} a_{k} z^{k}$. By (3), we have

$$
K_{r, t}(T)=\sum_{n \geq 0} r^{n} e^{i n t} T^{* n}+\sum_{n \geq 0} r^{n} e^{-i n t} T^{n}-I d .
$$


Since $\int_{0}^{2 \pi} e^{i l t} d t=0$ for $l \in \mathbb{Z} \backslash\{0\}$, we get:

$$
\begin{aligned}
\int_{0}^{2 \pi} p\left(e^{i t}\right) K_{r, t}(T) d t & =\sum_{k=0}^{N} \int_{0}^{2 \pi} a_{k} r^{k} T^{k} d t \\
& =2 \pi \sum_{k=0}^{N} a_{k} r^{k} T^{k} \\
& =2 \pi p(r T) .
\end{aligned}
$$

Moreover, using the operator-valued Poisson kernel, there is also another way to characterize the fact that an operator $T \in \mathcal{L}(\mathcal{H})$ is contractive.

Lemma 2.4. $\|T\| \leq 1 \Longleftrightarrow \sigma(T) \subset \overline{\mathbb{D}}$ and $K_{r, t}(T) \geq 0$ for all $r e^{i t} \in \mathbb{D}$.

Proof: $\quad$ Suppose that $T \in \mathcal{L}(\mathcal{H})$ is such that $\sigma(T) \subset \overline{\mathbb{D}}$ (automatically satisfied if $\|T\| \leq 1)$. Recall that $K_{r, t}(T) \geq 0$ if and only if $\left\langle K_{r, t}(T) h, h\right\rangle \geq 0$ for all $h \in \mathcal{H}$. Then, using (1), we have

$$
\left\langle K_{r, t}(T) h, h\right\rangle=\left\langle\left(I d-r^{2} T^{*} T\right) \tilde{h}, \tilde{h}\right\rangle=\|\tilde{h}\|^{2}-r^{2}\|T \tilde{h}\|^{2}
$$

with $\tilde{h}=\left(I d-r e^{-i t} T\right)^{-1} h$. The surjectivity of $\left(I d-r e^{-i t} T\right)^{-1}$ implies that $K_{r, t}(T) \geq 0$ if and only if $\|\tilde{h}\|^{2}-r^{2}\|T \tilde{h}\|^{2} \geq 0$ for all $\tilde{h} \in \mathcal{H}$. Now the assertion of the lemma is clear.

Now, let us present some nice applications of Lemma 2.3 and Lemma 2.4. The first one is a dilation-free proof of the well-known von Neumann's inequality discovered by Heinz [30]. This proof is reproduced in Section 153 of [43]. For a more recent account, see also [35]. Recall that von Neumann's inequality characterizes the Hilbert spaces among the complex Banach spaces. Indeed, it is proved in [29] that if $X$ is a complex Banach space such that the von Neumann's inequality is true for all contractions $T \in \mathcal{L}(X)$, then $X$ is necessarily a complex Hilbert space.

Proposition 2.5. Whenever $\|T\| \leq 1$, then

$$
\|p(T)\| \leq\|p\|_{\infty}=\sup _{z \in \mathbb{D}}|p(z)| \quad\left(p \in \mathbb{C}[z]_{\mid \overline{\mathbb{D}}}\right) .
$$


Proof: $\quad$ Let $x, y \in \mathcal{H}$. By Lemma 2.3, we have:

$$
\langle p(r T) x, y\rangle=\frac{1}{2 \pi} \int_{0}^{2 \pi} p\left(e^{i t}\right)\left\langle K_{r, t}(T) x, y\right\rangle d t \quad \text { for } r \in[0,1) .
$$

Using Lemma 2.4, we have also

$$
\left\langle K_{r, t}(T) x, y\right\rangle=\left\langle\tilde{x}_{r, t}, \tilde{y}_{r, t}\right\rangle,
$$

where $\tilde{x}_{r, t}=\sqrt{K_{r, t}(T)} x$ and $\tilde{y}_{r, t}=\sqrt{K_{r, t}(T)} y$. Therefore we get:

$$
|\langle p(r T) x, y\rangle| \leq \frac{\|p\|_{\infty}}{2 \pi} \int_{0}^{2 \pi}\left|\left\langle\tilde{x}_{r, t}, \tilde{y}_{r, t}\right\rangle\right| d t .
$$

Now, applying twice the Cauchy-Schwarz inequality, we get:

$$
\begin{aligned}
\int_{0}^{2 \pi}\left|\left\langle\tilde{x}_{r, t}, \tilde{y}_{r, t}\right\rangle\right| d t & \leq \int_{0}^{2 \pi}\left\|\tilde{x}_{r, t}\right\|\left\|\tilde{y}_{r, t}\right\| d t \\
& \leq\left(\int_{0}^{2 \pi}\left\|\tilde{x}_{r, t}\right\|^{2} d t\right)^{1 / 2}\left(\int_{0}^{2 \pi}\left\|\tilde{y}_{r, t}\right\|^{2} d t\right)^{1 / 2} .
\end{aligned}
$$

Using Lemma 2.3 with $p$ identically equal to 1 , we obtain:

$$
\int_{0}^{2 \pi}\left\|\tilde{x}_{r, t}\right\|^{2} d t=\int_{0}^{2 \pi}\left\langle K_{r, t}(T) x, x\right\rangle d t=2 \pi\|x\|^{2} .
$$

Finally we get

$$
|\langle p(r T) x, y\rangle| \leq\|p\|_{\infty}\|x\|\|y\|,
$$

which implies that $\|p(r T)\| \leq\|p\|_{\infty}$. Since

$$
\|p(r T)-p(T)\| \leq \sum_{k=0}^{N}\left(1-r^{k}\right)\left|a_{k}\right| \text { for } p(z)=\sum_{k=0}^{N} a_{k} z^{k},
$$

in particular, we obtain $\lim _{r \rightarrow 1}\|p(r T)\|=\|p(T)\|$ and therefore $\|p(T)\| \leq\|p\|_{\infty}$.

The second application will be used extensively in the next sections.

Proposition 2.6. Let $\|T\| \leq 1$. For all $x, y \in \mathcal{H}$, there exists a unique complex measure $\mu_{x, y}^{T} \in \mathcal{M}(\mathbb{T})$ such that

$$
\left\langle K_{r, t}(T) x, y\right\rangle=\frac{1}{2 \pi} \int_{0}^{2 \pi} P_{r}(\theta-t) d \mu_{x, y}^{T}(\theta)
$$

and there exists a unique function in $L^{1}$, denoted by $x^{T} \cdot y$ such that

$$
\left(x^{T} \cdot y\right)\left(e^{i t}\right)=\lim _{r \rightarrow 1^{-}}\left\langle K_{r, t}(T) x, y\right\rangle \text { and }\left\|x^{T} \cdot y\right\|_{1} \leq\|x\|\|y\| .
$$


Proof: $\quad$ For $z=r e^{i t} \in \mathbb{D}$, set $\varphi(z)=\left\langle K_{r, t}(T) x, y\right\rangle$. By (3), we have:

$$
\varphi(z)=\sum_{n \geq 0}\left\langle T^{* n} x, y\right\rangle z^{n}+\sum_{n \geq 0}\left\langle T^{n} x, y\right\rangle \bar{z}^{n}-\langle x, y\rangle,
$$

and thus $\varphi$ is an harmonic function on $\mathbb{D}$. In the proof of Proposition 2.5 , we have seen that

$$
\int_{0}^{2 \pi}\left|\left\langle K_{r, t}(T) x, y\right\rangle\right| d t \leq 2 \pi\|x\|\|y\| .
$$

Therefore we get

$$
\sup _{0 \leq r<1} \int_{0}^{2 \pi}\left|\varphi\left(r e^{i t}\right)\right| d t \leq 2 \pi\|x\|\|y\|<\infty .
$$

Applying Theorem A, there exists a unique complex measure $\mu_{x, y}^{T}$ on $\mathbb{T}$ such that

$$
\varphi\left(r e^{i t}\right)=\frac{1}{2 \pi} \int_{0}^{2 \pi} P_{r}(\theta-t) d \mu_{x, y}^{T}(\theta)
$$

and moreover, $\lim _{r \rightarrow 1} \varphi\left(r e^{i t}\right)$ is well-defined almost everywhere on $\mathbb{T}$ and is equal to the $L^{1}$-function denoted by $x^{T} \cdot y$ and called the Radon-Nikodym derivative of the elementary measure $\mu_{x, y}^{T}$. By Theorem A, we have also $\left\|\mu_{x, y}^{T}\right\|=\sup _{0 \leq r<1} \int_{0}^{2 \pi}\left|\varphi\left(r e^{i t}\right)\right| d t$. Since $\left\|x^{T} \cdot y\right\|_{1} \leq \frac{1}{2 \pi}\left\|\mu_{x, y}^{T}\right\|$, by (4), we obtain $\left\|x^{T} \cdot y\right\|_{1} \leq\|x\|\|y\|$.

Remark 2.7. Since $K_{r, t}(T)^{*}=K_{r, t}(T)$ and $K_{r, t}\left(T^{*}\right)=K_{r,-t}(T)$, we get $x^{T} \cdot y=\overline{y^{T} \cdot x}$, and $\left(x^{T^{*}} \cdot y\right)\left(e^{i t}\right)=\left(x^{T} \cdot y\right)\left(e^{-i t}\right)$ and therefore $\left(y^{T^{*}} x\right)\left(e^{i t}\right)=\overline{\left(x^{T} \cdot y\right)\left(e^{-i t}\right)}$.

The last result of this section concerns the possibility of extending analytically the density $x^{T} \cdot y$ (Lemma 5.1 in [16]).

Lemma 2.8. Let $T \in \mathcal{L}(\mathcal{H})$ be a contraction such that $\Gamma(T):=$ $\sigma(T) \cap \mathbb{T} \neq \mathbb{T}$. For any $x, y \in \mathcal{H}$ and any closed arc $I \in \mathbb{T} \backslash \Gamma(T)$, the function $x^{T} \cdot y$ extends analytically in a neighbourhood of $I$.

Proof: $\quad$ By (1), for $e^{i t} \notin \Gamma(T)$, we get exactly

$$
\left(x^{T} \cdot y\right)\left(e^{i t}\right)=\left\langle\left(I d-e^{i t} T^{*}\right)^{-1}\left(I d-T^{*} T\right)\left(I d-e^{-i t} T\right)^{-1} x, y\right\rangle .
$$

Hence, the function defined by

$$
z \longmapsto z\left\langle\left(I d-z T^{*}\right)^{-1}\left(I d-T^{*} T\right)(z I d-T)^{-1} x, y\right\rangle
$$


is an analytic extension of $x^{T} \cdot y$ in a neighbourhood of $I$.

\section{The $H^{\infty}$-Functional Calculus for an Absolutely Continuous Contraction}

A reformulation of von Neumann's inequality is the following: $\Phi_{T}: \mathbb{C}[z]_{\mid \overline{\mathbb{D}}} \rightarrow \mathcal{L}(\mathcal{H})$ defined by $\Phi_{T}(p)=p(T)$ is norm continuous $\left(\left\|\Phi_{T}\right\| \leq 1\right)$ and thus it extends continuously to $\widetilde{\Phi_{T}}$ (with $\left\|\widetilde{\Phi_{T}}\right\| \leq 1$ ) on $A(\mathbb{D})$, the disc algebra, defined by:

$$
A(\mathbb{D})=\left(\mathbb{C}[z]_{\mid \overline{\mathbb{D}}}\right)^{-\|\cdot\|_{\infty}}=C(\overline{\mathbb{D}}) \cap \operatorname{Hol}(\mathbb{D}) .
$$

In other words,

$$
\widetilde{\Phi_{T}}(f)=\lim _{n \rightarrow \infty} p_{n}(T) \text { (norm limit) }
$$

where $\left(p_{n}\right)_{n \geq 1}$ is a sequence of polynomials that converges uniformly to $f$ on $\mathbb{D}$.

Proposition 3.1. Let $f \in A(\mathbb{D})$ and $T \in \mathcal{L}(\mathcal{H})$ be a contraction. For all $x, y \in \mathcal{H}$, there exists a unique complex measure $\mu_{x, y}^{T}$ on $\mathbb{T}$ such that

$$
\langle f(T) x, y\rangle=\frac{1}{2 \pi} \int_{0}^{2 \pi} f\left(e^{i t}\right) d \mu_{x, y}^{T}(t)
$$

and

$$
\left\langle K_{r, t}(T) x, y\right\rangle=\frac{1}{2 \pi} \int_{0}^{2 \pi} P_{r}(\theta-t) d \mu_{x, y}^{T}(\theta) .
$$

Proof: $\quad$ Let $\mu_{x, y}^{T}$ be the measure defined in Proposition 2.6. It remains to check that $\langle f(T) x, y\rangle=\frac{1}{2 \pi} \int_{0}^{2 \pi} f\left(e^{i t}\right) d \mu_{x, y}^{T}(t)$. Let $f$ be a function in $A(\mathbb{D})$ and let $\left(p_{n}\right)_{n \geq 1}$ be a sequence of polynomials that converges uniformly to $f$ on $\mathbb{D}$. By definition, we have:

$$
\lim _{n \rightarrow \infty}\left\|f(r T)-p_{n}(r T)\right\|=0
$$

By Lemma 2.3, we have also

$$
p_{n}(r T)=\frac{1}{2 \pi} \int_{0}^{2 \pi} p_{n}\left(e^{i t}\right) K_{r, t}(T) d t .
$$

Since $\lim _{n \rightarrow \infty}\left\|p_{n}-f\right\|_{\infty}=0$, it follows that

$$
f(r T)=\frac{1}{2 \pi} \int_{0}^{2 \pi} f\left(e^{i t}\right) K_{r, t}(T) d t
$$


and therefore

$$
\langle f(r T) x, y\rangle=\frac{1}{2 \pi} \int_{0}^{2 \pi} f\left(e^{i t}\right)\left\langle K_{r, t}(T) x, y\right\rangle d t .
$$

Now, since $\left\langle K_{r, t}(T) x, y\right\rangle=\frac{1}{2 \pi} \int_{0}^{2 \pi} P_{r}(\theta-t) d \mu_{x, y}^{T}(\theta)$, by Fubini's theorem, and the fact that $\frac{1}{2 \pi} \int_{0}^{2 \pi} f\left(e^{i t}\right) P_{r}(\theta-t) d t=f\left(r e^{i \theta}\right)$, it follows that:

$$
\langle f(r T) x, y\rangle=\int_{0}^{2 \pi} f\left(r e^{i \theta}\right) d \mu_{x, y}^{T}(\theta) .
$$

Define $f_{r} \in A(\mathbb{D})$ by $f_{r}(z)=f(r z)$. Since $\lim _{r \rightarrow 1}\left\|f_{r}-f\right\|_{\infty}=0$, the continuity of the $A(\mathbb{D})$-functional calculus $\widetilde{\Phi_{T}}$, implies that

$$
\langle f(T) x, y\rangle=\frac{1}{2 \pi} \int_{0}^{2 \pi} f\left(e^{i t}\right) d \mu_{x, y}^{T}(t) .
$$

Remark 3.2. Let $T$ be a contraction, and let $f, g \in A(\mathbb{D})$. Since $f(T) g(T)=(f g)(T)$, it follows that $f d \mu_{x, y}^{T}=d \mu_{f(T) x, y}^{T}$.

Now, we would like to extend the $A(\mathbb{D})$-functional calculus into a $H^{\infty}$-functional calculus. To that aim, we need to introduce the next definition.

Definition 3.3. A contraction $T \in \mathcal{L}(\mathcal{H})$ is said to be absolutely continuous if for all $x, y \in \mathcal{H}$, the elementary spectral measure $\mu_{x, y}^{T}$ satisfying

$$
\left\langle K_{r, t}(T) x, y\right\rangle=\frac{1}{2 \pi} \int_{0}^{2 \pi} P_{r}(\theta-t) d \mu_{x, y}^{T}(\theta)
$$

is absolutely continuous with respect to Lebesgue measure. In other words, using the notation of Proposition 2.6, $T$ is absolutely continuous (a.c.) if $d \mu_{x, y}^{T}(t)=\left(x^{T} \cdot y\right)\left(e^{i t}\right) d t$ for all $x, y \in \mathcal{H}$.

Recall the canonical decomposition of a contraction proved in [45] (see also [46], Chap. I.3).

Theorem B. Every contraction $T \in \mathcal{L}(\mathcal{H})$ has a unique decomposition of the form $T=T_{0} \oplus T_{1}$ where $T_{0}$ is completely nonunitary and $T_{1}$ is unitary. 
Using dilation theory, B. Sz.-Nagy and C. Foias ([46], Chap. III.2) proved the following characterization of absolutely continuous contractions.

Theorem C. Let $T \in \mathcal{L}(\mathcal{H})$ be a contraction. Then $T$ is absolutely continuous if and only if the unitary part of $T$ has an absolutely continuous spectral measure.

Remark 3.4. In particular, if $T$ is a completely nonunitary contraction, then $T$ is absolutely continuous.

Proposition 3.5. Let $T \in \mathcal{L}(\mathcal{H})$ be an absolutely continuous contraction (a.c.c.). Then $T$ has a $w^{*}-w^{*}$ contractive and multiplicative $H^{\infty}$-functional calculus $\Psi_{T}$ extending its $A(\mathbb{D})$-functional calculus and defined as follows:

$f(T):=\Psi_{T}(f), f \in H^{\infty}$ and for all $x, y \in \mathcal{H}$,

$$
\langle f(T) x, y\rangle=\frac{1}{2 \pi} \int_{0}^{2 \pi} f^{*}\left(e^{i t}\right)\left(x^{T} \cdot y\right)\left(e^{i t}\right) d t,
$$

where $\left(x^{T} \cdot y\right)=\lim _{r \rightarrow 1^{-}}\left\langle K_{r, t}(T) x, y\right\rangle$ and where $f^{*} \in L^{\infty}$ is defined almost everywhere by $f^{*}\left(e^{i t}\right)=\lim _{r \rightarrow 1} f\left(r e^{i t}\right)$.

Remark 3.6. The set of absolutely continuous contractions can also be defined as the set of contractions admitting an $H^{\infty}$-functional calculus $\Psi_{T}$ extending its $A(\mathbb{D})$-functional calculus. Moreover $T$ is an a.c.c. if and only if $T^{*}$ is an a.c.c.

We have an explicit expression of the Fourier coefficients of the density of the elementary spectral measures.

Lemma 3.7. Let $T \in \mathcal{L}(\mathcal{H})$ be an a.c.c. For all $x, y \in \mathcal{H}$, we have:

$$
\widehat{x^{T} \cdot y}(n)=\left\{\begin{array}{lll}
\left\langle T^{* n} x, y\right\rangle & \text { if } & n \geq 0 \\
\left\langle T^{|n|} x, y\right\rangle & \text { if } & n \leq 0
\end{array}\right.
$$

Proof: Using (5) with $f(z)=z^{n}, n \geq 0$, we get:

$$
\left\langle T^{n} x, y\right\rangle=\frac{1}{2 \pi} \int_{0}^{2 \pi} e^{i n t}\left(x^{T} \cdot y\right)\left(e^{i t}\right) d t=\widehat{x^{T} \cdot y}(-n) .
$$

Moreover, replacing $T$ by $T^{*}$, we have also

$$
\left\langle T^{* n} x, y\right\rangle=\frac{1}{2 \pi} \int_{0}^{2 \pi} e^{i n t}\left(x^{T^{*}} y\right)\left(e^{i t}\right) d t .
$$


Now, by Remark 2.7, we get:

$$
\begin{aligned}
\left\langle T^{* n} x, y\right\rangle & =\frac{1}{2 \pi} \int_{0}^{2 \pi} e^{i n t}\left(x^{T} \cdot y\right)\left(e^{-i t}\right) d t \\
& =\frac{1}{2 \pi} \int_{0}^{2 \pi} e^{-i n t}\left(x^{T} \cdot y\right)\left(e^{i t}\right) d t=\widehat{x^{T} \cdot y}(n) .
\end{aligned}
$$

Remark 3.8. Obviously, when $T \in \mathcal{L}(\mathcal{H})$ is an a.c.c., $T$ has also a $w^{*}-w^{*}$ contractive $L^{\infty}$-functional calculus (extending its $H^{\infty}$ functional calculus) defined as follows: for $f \in L^{\infty}$ and for all $x, y \in \mathcal{H}$,

$$
\langle f(T) x, y\rangle=\frac{1}{2 \pi} \int_{0}^{2 \pi} f\left(e^{i t}\right)\left(x^{T} \cdot y\right)\left(e^{i t}\right) d t
$$

where $\left(x^{T} \cdot y\right)=\lim _{r \rightarrow 1^{-}}\left\langle K_{r, t}(T) x, y\right\rangle$. This $L^{\infty}$-functional calculus is multiplicative if and only if $T$ is unitary, which is a very restrictive condition.

\section{Applications to the Invariant Subspace Problem}

Let $X$ be a separable, complex and infinite dimensional Banach space. Denote by $\mathcal{L}(X)$ the algebra of all bounded and linear operators from $X$ to $X$, and for $T \in \mathcal{L}(X)$ denote by $\operatorname{Lat}(T)$ the lattice of all the closed subspaces $\mathcal{M}$ of $X$ such that $T \mathcal{M} \subset \mathcal{M}$. We say that an operator $T \in \mathcal{L}(X)$ is transitive if $\operatorname{Lat}(T)=\{X,\{0\}\}$.

P. Enflo [26, 27], C. Read [37, 38, 39, 40, 41, 42], B. Beauzamy $[2,3]$ have constructed transitive operators $T \in \mathcal{L}(X)$, where $X$ is a nonreflexive Banach space $\left(\ell^{1}, c_{0}, \ldots\right)$, with $\sigma(T)$ very rich or reduced to a singleton.

The invariant subspace problem (ISP) is the following:

\section{When $X$ is a Hilbert space $\mathcal{H}$, does there exist a transitive operator $T \in \mathcal{L}(\mathcal{H})$ ?}

There is a huge number of positive results concerning the invariant subspace problem, using a wide range of techniques (cf. [36, 19]). One of them is the very nice result of V. I. Lomonosov [33] who proved the next theorem: 
Theorem D. Let $T \in \mathcal{L}(X)$ be such that there exists $K \neq 0$ compact satisfying $T K=K T$. Then $T$ is not transitive.

Another nice result, whose interest lies mostly in an approximation process which has led to a large number of applications, is the following [10].

Theorem E. Let $T \in \mathcal{L}(\mathcal{H})$ be a subnormal operator (i.e., the restriction to an invariant subspace of an operator $N$ such that $N N^{*}=$ $N^{*} N$ ). Then $T$ is not transitive.

In 1991, V. I. Lomonosov [34] conjectured that if $T \in \mathcal{L}(X)$ where $X$ is a Banach space, then $T^{*}$ is not transitive.

For $T \in \mathcal{L}(\mathcal{H})$, since $T \mathcal{M} \subset \mathcal{M}$ if and only if $T^{*} \mathcal{M}^{\perp} \subset \mathcal{M}^{\perp}$, a proof of the Lomonosov's conjecture would clearly imply a positive answer to the invariant subspace problem.

Concerning (ISP), since a nontrivial unitary operator has nontrivial invariant subspaces, one may always assume that $T \in \mathcal{L}(\mathcal{H})$ is a contraction which is completely nonunitary, and therefore admits an $H^{\infty}$-functional calculus.

4.1. S. Brown's method. Our aim is now to give an idea of S. Brown's method and to show the link with the two previous sections.

The starting point of S. Brown's method is the following lemma.

Lemma 4.1. Let $T \in \mathcal{L}(\mathcal{H}),\|T\| \leq 1$ and $\lambda \in \mathbb{D}$. Suppose that there exist $x, y \in \mathcal{H}$ such that

$$
\langle x, y\rangle=1 \text { and }\left\langle T^{n} x, y\right\rangle=\lambda^{n} \text { for } n \geq 1 .
$$

Then $T$ has a nontrivial invariant subspace.

Proof: $\quad$ Let $\mathcal{M}$ the closed linear hull of $\left\{(T-\lambda I d) T^{n} x: n \geq 0\right\}$. Then $T \mathcal{M} \subset \mathcal{M}$. The equality (7) implies that $y \perp \mathcal{M}$ with $y \neq 0$. Then $\mathcal{M}$ or $\mathbb{C} x$ is a nontrivial invariant subspace.

In view of the previous section, one can reformulate Lemma 4.1. We have seen that if $T \in \mathcal{L}(\mathcal{H})$ is an a.c.c., for all $x, y \in \mathcal{H}$ there exists a unique function $x^{T} \cdot y \in L^{1}$ such that

$$
\begin{cases}\left(T^{n} x, y\right)=\widehat{\left(x^{T} \cdot y\right)}(-n), & n \geq 0 \\ \left(T^{* n} x, y\right)=\widehat{\left(x^{T} \cdot y\right)}(n), & n>0 .\end{cases}
$$


For $\lambda \in \mathbb{D}$, set $P_{\lambda}\left(e^{i t}\right)=\sum_{n \geq 0} \lambda^{n} e^{-i n t}+\sum_{n \geq 1} \overline{\lambda^{n}} e^{i n t}$.

A reformulation of S. Brown's starting point is:

If there are $x, y \in \mathcal{H}, \lambda \in \mathbb{D}$ such that $\left[x^{T} \cdot y\right]_{L^{1} / H_{0}^{1}}=\left[P_{\lambda}\right]_{L^{1} / H_{0}^{1}}$, then $T$ is not transitive.

What is now called the S. Brown's approximation process is the next result [7].

Theorem F. Let E, $F$ and $G$ be complex Banach spaces, and let $\varphi: E \times F \rightarrow G$ be a bilinear or a sesquilinear map. Suppose that there exists $K>0$ such that for all $z \in G$, there exists a sequence $\left(x_{n}, y_{n}\right)_{n}$ in $E \times F$ satisfying:

$$
\begin{cases}\lim _{n \rightarrow \infty}\left\|\varphi\left(x_{n}, y_{n}\right)-z\right\|=0 & \\ \left\|x_{n}\right\|\left\|y_{n}\right\| \leq K\|z\|, & n \geq 1 \\ \lim _{n \rightarrow \infty}\left(\left\|\varphi\left(x, y_{n}\right)\right\|+\left\|\varphi\left(x_{n}, y\right)\right\|\right)=0, & x \in E, y \in F .\end{cases}
$$

Then for all $z \in G$ there exists $(u, v) \in E \times F$ such that $\varphi(u, v)=z$. In other words, $\varphi$ is surjective.

This approximation process has been extensively used in dual algebra theory (see for example [6]) in the particular case where $T \in \mathcal{L}(\mathcal{H})$ is an a.c.c., $E=F=\mathcal{H}, G=L^{1}$ or $L^{1} / H_{0}^{1}$ and where $\varphi$ is the sesquilinear map defined by $\varphi(x, y)=x^{T} \cdot y$ or $\varphi(x, y)=\left[x^{T} \cdot y\right]_{L^{1} / H_{0}^{1}}$.

4.2. The class $\mathbb{A}$. Previously, we have mentioned that an a.c.c. $T \in \mathcal{L}(\mathcal{H})$ has a contractive $H^{\infty}$-functional calculus $\Psi_{T}$. The case where $\Psi_{T}$ is an isometry is of particular interest because of Apostol's result [1].

Theorem G. Let $T \in \mathcal{L}(\mathcal{H})$ be a contraction such that $\sigma(T) \supset \mathbb{T}$. Then either $T$ has a nontrivial invariant subspace or $T$ is an a.c.c. in the class $\mathbb{A}$, that is whose $H^{\infty}$-functional calculus $\Psi_{T}$ is isometric.

We will present now some sufficient conditions for a contraction to be in the class $\mathbb{A}$. To that aim, we introduce the following definition.

Definition 4.2. A subset $\Gamma$ of $\mathbb{D}$ is a dominating set of $\mathbb{D}$ (or a dominating set for $H^{\infty}$ ) if, for every $f \in H^{\infty}$, we have:

$$
\|f\|_{\infty}=\sup \{|f(\lambda)|: \lambda \in \Gamma\} .
$$


The dominating sets of $\mathbb{D}$ were characterized in [9], Theorem 3, as follows: a subset $\Gamma$ of $\mathbb{D}$ is a dominating set of $\mathbb{D}$ if and only if almost every point $\xi \in \mathbb{T}$ is a nontangential limit point of $\Gamma$ (that is, $\xi$ is the limit of a sequence in $\Gamma$ that lies inside some proper angular opening with vertex at $\xi$ ).

Lemma 4.3. If $T$ is an a.c.c. such that $\sigma(T)$ is a dominating set of $\mathbb{D}$, then $T \in \mathbb{A}$.

Proof: $\quad$ Recall that if $T$ is an a.c.c., its $H^{\infty}$-functional calculus is contractive, and thus $\|f(T)\| \leq\|f\|_{\infty}, f \in H^{\infty}$. Using the spectral mapping inclusion $f(\sigma(T)) \subset \sigma(f(T))$ and the fact that $\|f(T)\| \geq$ $\sup \{|\beta|: \beta \in \sigma(f(T))\}$ we have $\sup \{|f(\lambda)|: \lambda \in \sigma(T) \cap \mathbb{D}\} \leq\|f(T)\|$. Therefore, if $\sigma(T)$ is a dominating set of $\mathbb{D}$, then $T \in \mathbb{A}$.

In [12], S. Brown, B. Chevreau and C. Pearcy proved that if $T$ is contraction such that $\mathbb{T} \subset \sigma(T)$, then $T$ has nontrivial invariant subspaces. A few months later, Bercovici [4] and Chevreau [23] proved independently that if $T \in \mathbb{A}$, then for all $f \in L^{1}$, there exist $x, y \in \mathcal{H}$ such that

$$
[f]_{L^{1} / H_{0}^{1}}=\left[x^{T} \cdot y\right]_{L^{1} / H_{0}^{1}}
$$

Thanks to Theorem $\mathrm{G}$, the existence of nontrivial invariant subspaces for a contraction $T$ such that $\mathbb{T} \subset \sigma(T)$ can be seen as a corollary of (8) applied with $f=P_{r, t}$ for $r e^{i t} \in \mathbb{D}$.

In 1988, Brown and Chevreau [11] proved that if $T \in \mathbb{A}$, then $T$ is reflexive. Recall that $T$ is reflexive if for all $B \in \mathcal{L}(\mathcal{H})$ satisfying Lat $(T) \subset \operatorname{Lat}(B)$, there exists $f \in H^{\infty}$ such that $B=f(T)$ (a trivial sufficient condition which ensures that $\operatorname{Lat}(T) \subset \operatorname{Lat}(B))$. Therefore, the class $\mathbb{A}$ is a class of contractions whose lattice of invariant subspaces is particularly rich.

4.3. Classes $\mathbb{A}_{n, m}$. In 1983, H. Bercovici, C. Foias and C. Pearcy [5] introduced subclasses of the class $\mathbb{A}$, defined as follows.

Definition 4.4. Let $1 \leq n, m \leq \aleph_{0}$. Then

$$
\begin{gathered}
\mathbb{A}_{n, m}=\left\{T \in \mathbb{A}: \forall\left(f_{p, q}\right) \subset L^{1}, \exists\left(x_{p}\right)_{0 \leq p<n},\left(y_{q}\right)_{0 \leq q<m} \subset \mathcal{H}\right. \\
\text { such that } \left.\left[f_{p, q}\right]_{L^{1} / H_{0}^{1}}=\left[x_{p}{ }^{T} \cdot y_{q}\right]_{L^{1} / H_{0}^{1}}\right\} .
\end{gathered}
$$


By (8), we have $\mathbb{A}=\mathbb{A}_{1,1}$ and by definition, we have the following scheme of inclusion for the classes $\mathbb{A}_{n, m}$.

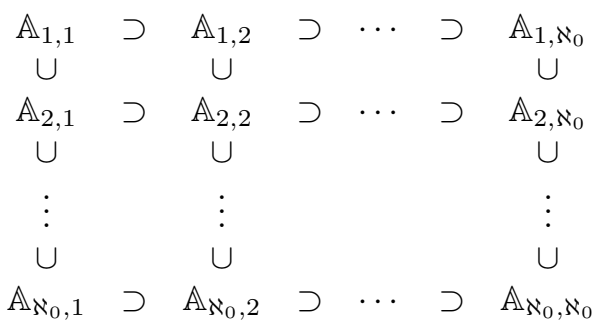

One can find characterizations and information about the classes $\mathbb{A}_{n, m}$ in $[6,16,17,20]$. The classes $\mathbb{A}_{n, m}$ have the property that the larger the indices $n$ and $m$ are, the richer the lattice of invariant subspaces is. Some of the properties of the classes $\mathbb{A}_{n, m}$ are presented in the next proposition.

Proposition 4.5. Let $0 \leq n, m \leq \aleph_{0}$ and $T \in \mathcal{L}(\mathcal{H})$ be an a.c.c.

(1) $T \in \mathbb{A}_{n, m}$ if and only if $T^{*} \in \mathbb{A}_{m, n}$.

(2) If $T \in \mathbb{A}_{n, n}$ then, for all $\lambda \in \mathbb{D}$, there is $\mathcal{M}_{\lambda} \in \operatorname{Lat}(T)$ such that $\operatorname{dim}\left(\mathcal{M}_{\lambda} \ominus(T-\lambda I d) \mathcal{M}_{\lambda}\right)=n$.

(3) If $T \in \mathbb{A}_{\aleph_{0}, \aleph_{0}}$, then Lat $(T)$ contains a sublattice isomorphic to the set of all closed subspaces of $\mathcal{H}$.

Proof: 1. This is an obvious consequence of the equality

$$
\left(y^{T^{*}} x\right)\left(e^{i t}\right)=\overline{\left(x^{T} \cdot y\right)\left(e^{-i t}\right)}
$$

(see Remark 2.7) and the fact that for all $f \in L^{1}$ there exists $g \in L^{1}$ such that $f\left(e^{i t}\right)=\overline{g\left(e^{-i t}\right)}$.

2. If $T \in \mathbb{A}_{n, n}$, taking $f_{p, p}=P_{r, t}\left(\right.$ with $\left.\lambda=r e^{i t}\right)$ and $f_{p, q}=0$ for $0 \leq p, q<n$ with $p \neq q$, there exist $\left(x_{p}\right)_{0 \leq p<n}$ and $\left(y_{q}\right)_{0 \leq q<n}$ in $\mathcal{H}$ such that

$$
\left\langle T^{k} x_{p}, y_{q}\right\rangle=\delta_{p, q} \lambda^{k} .
$$

Consider $\mathcal{M}_{\lambda}$ the invariant subspace for $T$ generated by $\left(x_{p}\right)_{0 \leq p<n}$. Then $\operatorname{dim}\left(\mathcal{M}_{\lambda} \ominus(T-\lambda I d) \mathcal{M}_{\lambda}\right)$ is necessarily at most $n$ and is at least $n$ because, by (9), the vectors $y_{q}^{\prime}$ equal to the orthogonal projection of $y_{q}$ onto $\mathcal{M}_{\lambda}$ are linearly independent and belong to the space $\mathcal{M}_{\lambda} \ominus(T-\lambda I d) \mathcal{M}_{\lambda}$.

3. If $T \in \mathbb{A}_{\aleph_{0}, \aleph_{0}}$, taking $f_{p, p}=P_{0,0}=1$ and $f_{p, q}=0$ for $0 \leq$ $p, q<\aleph_{0}$ with $p \neq q$, along the same lines of the proof of 2 ., one can 
prove that there exists $\mathcal{M} \in \operatorname{Lat} T$ such that $\operatorname{dim} \mathcal{M} \ominus T \mathcal{M}=\aleph_{0}$. Set $\mathcal{K}=\mathcal{M} \ominus T \mathcal{M}$. Obviously we have $P_{\mathcal{K}} T_{\mid \mathcal{K}}=0$, where $P_{\mathcal{K}}$ is the orthogonal projection of $\mathcal{H}$ onto $\mathcal{K}$. By construction, we have $\left\{\mathcal{K}^{\prime} \oplus T \mathcal{M}: \mathcal{K}^{\prime}\right.$ a closed subspace of $\left.\mathcal{K}\right\}$ contained in Lat $(T)$.

In what follows we will give an idea of the proof of the fact that classes $\mathbb{A}_{n, m}$ are distinct.

Let $L=\{z \in \mathbb{C}:(|z|=1, \Re(z) \leq 0) \quad$ or $\quad(\Re(z)=0,|\Im(z)| \leq 1)\}$. Thus $L$ is the boundary of the open left half unit disc; let $\mathbb{D}_{L}$ denote this left half disc (so $\mathbb{D}_{L}$ is the simply connected component of $\mathbb{C} \backslash L$ ). Put arc-length measure $\ell$ on $L$, and define $L^{2}(L, d \ell)$ to be the space of (equivalence classes of) square integrable complex functions on $L$. Now, define $E^{2}(L, d \ell)$ as the closure of the polynomials in $L^{2}(L, d \ell)$. Similarly, let us define

$$
R=\{z \in \mathbb{C}:|z|=1, \Re(z) \geq 0\} \cup\{z \in \mathbb{C}: \Re(z)=0,|\Im(z)| \leq 1)\} .
$$

So $R$ is the boundary of the open right half unit disc $\mathbb{D}_{R}$ and we define $L^{2}(R, d \ell)$ and $E^{2}(R, d \ell)$ in the analogous way.

Let $N_{L}$ be the (normal) operator of multiplication by $z$ on the space $L^{2}(L, d \ell)$, and $T_{L}$ its (subnormal) restriction to $E^{2}(L, d \ell)$. Let $N_{R}$ and $T_{R}$ be defined similarly relative to $R$.

Let $w=\phi(z)$ be a map that takes the disc to the left semi-disc $\mathbb{D}_{L}$; for example, $z=\phi^{-1}(w)=\left(w^{2}-2 w-1\right) /\left(w^{2}+2 w-1\right)$. Note that $\phi^{\prime}(z)=\left(w^{2}+2 w-1\right)^{2} /\left(4\left(w^{2}+1\right)\right)$. Then, we have an unitary equivalence between $T_{L}$ and the operator $M_{\phi}$ on $H^{2}$, where $M_{\phi}(f)=\phi f$.

The following formula will be of use in interpreting factorizations with $M_{\phi}$. In what follows $\phi: \mathbb{D} \rightarrow \mathbb{D}_{L}$ is a conformal bijection, and we shall choose it so that the left-hand $\operatorname{arc}\left[e^{i \pi / 2}, e^{3 i \pi / 2}\right]$ is mapped to itself (to achieve this, consider instead $\phi \circ \mu$ for a suitable Möbius map $\mu$ ).

Lemma 4.6. For $\pi / 2<t<3 \pi / 2$ and $f, g \in H^{2}(\mathbb{T})$, we have:

$$
\begin{aligned}
& f^{M_{\phi}} g\left(e^{i t}\right)=\frac{f \bar{g}\left(\phi^{-1}\left(e^{i t}\right)\right) e^{i t}}{\phi^{\prime}\left(\phi^{-1}\left(e^{i t}\right)\right) \phi^{-1}\left(e^{i t}\right)}+ \\
& \int_{-\pi / 2}^{\pi / 2} f\left(e^{i \theta}\right) \overline{g\left(e^{i \theta}\right)} \frac{1-\left|\phi\left(e^{i \theta}\right)\right|^{2}}{\left|1-e^{-i t} \phi\left(e^{i \theta}\right)\right|^{2}} d \theta,
\end{aligned}
$$

and the second term has an analytic extension to the left-hand half plane $\{\Re z<0\}$. 
Proof: Using (1), we have, for $\pi / 2<t<3 \pi / 2$,

$$
\begin{aligned}
f^{M_{\phi}} g\left(e^{i t}\right)= & \lim _{r \rightarrow 1^{-}} \int_{0}^{2 \pi} f\left(e^{i \theta}\right) \overline{g\left(e^{i \theta}\right)} \frac{1-r^{2}\left|\phi\left(e^{i \theta}\right)\right|^{2}}{\left|1-r e^{-i t} \phi\left(e^{i \theta}\right)\right|^{2}} d \theta \\
= & \lim _{r \rightarrow 1^{-}} \int_{\pi / 2}^{3 \pi / 2} f\left(e^{i \theta}\right) \overline{g\left(e^{i \theta}\right)} \frac{1-r^{2}}{\left|1-r e^{-i t} \phi\left(e^{i \theta}\right)\right|^{2}} d \theta \\
& \quad+\int_{-\pi / 2}^{\pi / 2} f\left(e^{i \theta}\right) \overline{g\left(e^{i \theta}\right)} \frac{1-\left|\phi\left(e^{i \theta}\right)\right|^{2}}{\left|1-e^{-i t} \phi\left(e^{i \theta}\right)\right|^{2}} d \theta .
\end{aligned}
$$

Define a new function $\psi: \mathbb{T} \rightarrow \mathbb{T}$ by

$$
\psi\left(e^{i \theta}\right)= \begin{cases}\phi\left(e^{i \theta}\right) & \text { if } \pi / 2<\theta<3 \pi / 2 \\ e^{i \theta} & \text { otherwise. }\end{cases}
$$

Then, since $\lim _{r \rightarrow 1^{-}} \frac{1-r^{2}}{\left|1-r e^{-i t} \psi\left(e^{i \theta}\right)\right|^{2}}=0$ for $\theta \in(0,2 \pi) \backslash\left[\frac{\pi}{2}, \frac{3 \pi}{2}\right]$, we get:

$$
\begin{aligned}
f^{M_{\phi}} g\left(e^{i t}\right)= & \lim _{r \rightarrow 1^{-}} \int_{0}^{2 \pi} f\left(e^{i \theta}\right) \overline{g\left(e^{i \theta}\right)} \frac{1-r^{2}}{\left|1-r e^{-i t} \psi\left(e^{i \theta}\right)\right|^{2}} d \theta \\
& \quad+\int_{-\pi / 2}^{\pi / 2} f\left(e^{i \theta}\right) \overline{g\left(e^{i \theta}\right)} \frac{1-\left|\phi\left(e^{i \theta}\right)\right|^{2}}{\left|1-e^{-i t} \phi\left(e^{i \theta}\right)\right|^{2}} d \theta \\
= & \frac{f \bar{g}\left(\phi^{-1}\left(e^{i t}\right)\right) e^{i t}}{\phi^{\prime}\left(\phi^{-1}\left(e^{i t}\right)\right) \phi^{-1}\left(e^{i t}\right)} \\
& \quad+\int_{-\pi / 2}^{\pi / 2} f\left(e^{i \theta}\right) \overline{g\left(e^{i \theta}\right)} \frac{1-\left|\phi\left(e^{i \theta}\right)\right|^{2}}{\left|1-e^{-i t} \phi\left(e^{i \theta}\right)\right|^{2}} d \theta,
\end{aligned}
$$

using a change of variables and the standard properties of the Poisson kernel. Note that the first term involves the value of $f \bar{g}$ at just one point of the left-hand semi-circle, and the second term involves values only on the right-hand semi-circle.

Since, for $e^{i t}$ on the unit circle,

$$
\begin{aligned}
\left|1-e^{-i t} w\right|^{-2} & =\left|e^{i t}-w\right|^{-2} \\
& =\left(e^{i t}-w\right)^{-1}\left(e^{-i t}-\bar{w}\right)^{-1} \\
& =\frac{e^{i t}}{\left(e^{i t}-w\right)\left(1-\bar{w} e^{i t}\right)},
\end{aligned}
$$

it is clear that the second term has an analytic extension to the left-hand half plane $\{\Re z<0\}$.

The previous lemma will be useful for the proof of the next result. 
Theorem 4.7. We have $T_{L} \oplus T_{R}^{*} \in \mathbb{A} \backslash\left(\mathbb{A}_{1,2} \cup \mathbb{A}_{2,1}\right)$.

Proof: Clearly $\sigma\left(T_{L} \oplus T_{R}^{*}\right)=\overline{\mathbb{D}}$, which, using Lemma 4.3 is a sufficient condition for membership in $\mathbb{A}$. Using the first assertion of Proposition 4.5, it is sufficient to prove that $M_{\phi} \oplus T_{R}^{*} \notin \mathbb{A}_{2,1}$. Let $\delta=1 / 2$ and $C_{\delta}=\{z \in \mathbb{C}: \Re z<-\delta\}$. Now consider the function $\gamma$ defined on $\mathbb{T}$ by

$$
\gamma\left(e^{i t}\right)=\frac{e^{i t}}{\phi^{\prime}\left(\phi^{-1}\left(e^{i t}\right)\right) \phi^{-1}\left(e^{i t}\right)} .
$$

Take $\Omega_{1}$ and $\Omega_{2}$ to be closed subarcs of $C_{\delta} \cap \mathbb{T}$ such that $\ell\left(\Omega_{1}^{c} \cap \Omega_{2}^{c} \cap\right.$ $\left.C_{\delta}\right)>0, \ell\left(\Omega_{1} \cap \Omega_{2}\right)=0$, and $\ell\left(\Omega_{j}\right)>0$ for $j=1$, 2 . Suppose that $M_{\phi} \oplus T_{R}^{*} \in \mathbb{A}_{2,1}$. It follows that there exist functions $f_{1}, f_{2}, g$ in $H^{2}$ and $x_{1}, x_{2}, y$ in $E^{2}(R, d \ell)$ such that:

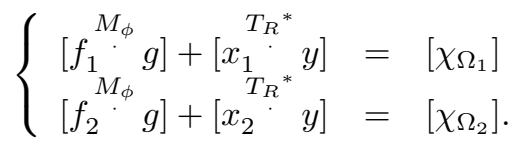

Since $\sigma\left(T_{R}^{*}\right) \cap \mathbb{T}=R \cap \mathbb{T}$, it follows from the proof of Lemma 2.8 that $T_{R}{ }^{*} T_{R}{ }^{*}$

$x_{1}{ }^{R} y$ and $x_{2}{ }^{*} \quad y$ have analytic extensions to $C_{\delta}$. Using Lemma 4.6 there exist $h_{1}$ and $h_{2}$ in $H^{2}$ and $k_{1}, k_{2}$ analytic on $C_{\delta}$, such that, on $C_{\delta} \cap \mathbb{T}$,

$\left\{\begin{array}{l}\chi_{\Omega_{1}}\left(e^{i t}\right)+e^{i t} h_{1}\left(e^{i t}\right)=f_{1}\left(\phi^{-1}\left(e^{i t}\right)\right) \overline{g\left(\phi^{-1}\left(e^{i t}\right)\right)} \gamma\left(e^{i t}\right)+k_{1}\left(e^{i t}\right) \\ \chi_{\Omega_{2}}\left(e^{i t}\right)+e^{i t} h_{2}\left(e^{i t}\right)=f_{2}\left(\phi^{-1}\left(e^{i t}\right)\right) \overline{g\left(\phi^{-1}\left(e^{i t}\right)\right)} \gamma\left(e^{i t}\right)+k_{2}\left(e^{i t}\right) .\end{array}\right.$

On $\Omega_{1}^{c} \cap \Omega_{2}^{c} \cap C_{\delta}$ we have

$\left[e^{i t} h_{1}\left(e^{i t}\right)-k_{1}\left(e^{i t}\right)\right] f_{2}\left(\phi^{-1}\left(e^{i t}\right)\right)=\left[e^{i t} h_{2}\left(e^{i t}\right)-k_{2}\left(e^{i t}\right)\right] f_{1}\left(\phi^{-1}\left(e^{i t}\right)\right)$,

and hence the same inequality holds on $C_{\delta} \cap \mathbb{T}$ since both sides of the equation are $E^{1}$ (Hardy class) functions on $C_{\delta} \cap \mathbb{D}$. Multiplying the first of the above pair of identities by $f_{2}\left(\phi^{-1}\left(e^{i t}\right)\right)$ and the second by $f_{1}\left(\phi^{-1}\left(e^{i t}\right)\right)$ and subtracting, we see that

$$
\chi_{\Omega_{1}}\left(e^{i t}\right) f_{2}\left(\phi^{-1}\left(e^{i t}\right)\right)=\chi_{\Omega_{2}}\left(e^{i t}\right) f_{1}\left(\phi^{-1}\left(e^{i t}\right)\right) \quad \text { on } \quad C_{\delta} \cap \mathbb{T} \text {, }
$$

which implies that $f_{1}$ and $f_{2}$ are identically zero, since they vanish on subsets of positive measure. This is absurd as $\chi_{\Omega_{1}}$ and $\chi_{\Omega_{2}}$ are not the restrictions of analytic functions.

For every pair of positive integers $n$ and $m$, denote by $T_{L}^{(n)} \oplus T_{R}^{*(m)}$ the operator given by the direct sum of $n$ copies of $T_{L}$ and $m$ copies of $T_{R}^{*}$. 
One can extend the methods of this section to obtain the following result (Theorem 5.2 in [21]), establishing Conjecture 3.5 of [28],

which provides a constructive proof of the fact that the classes $\mathbb{A}_{n, m}$ are distinct. Recall that a non constructive proof was given in [32].

Theorem 4.8. We have $T_{L}^{(n)} \oplus T_{R}^{*(m)} \in \mathbb{A}_{n, m} \backslash\left(\mathbb{A}_{n+1,1} \cup \mathbb{A}_{1, m+1}\right)$.

\section{Applications in Harmonic Analysis}

Most of the results of this section comes from [21, 22]. The degree of a finite Blaschke product $b$ is defined to be the cardinality of its zero set $Z \operatorname{ero}(b)$ (taking into account multiplicity). The next result [21] provides a link between the density of the elementary spectral measure associated with $T$ and that associated with $b(T)$.

Theorem 5.1. Let $T \in \mathcal{L}(\mathcal{H})$ be an a.c.c. and let $b$ be a finite Blaschke product of degree $d$. Then

$$
\left(x^{b(T)} y\right)\left(e^{i t}\right)=\sum_{j=1}^{d} \frac{\left(x^{T} \cdot y\right)\left(\xi_{j}\right)}{\left|b^{\prime}\left(\xi_{j}\right)\right|} \quad \text { a.e. }
$$

where $\xi_{1}, \ldots, \xi_{d}$ are the solutions of $b(z)=e^{i t}$.

Proof: $\quad$ We may partition $\mathbb{T}$ into intervals $J_{1}, \ldots, J_{d}$, each of which is mapped onto $\mathbb{T}$ by $b$ (see, for example, [15]). Then, we get:

$$
\begin{aligned}
\left(x^{b(T)} y\right)\left(e^{i t}\right)= & \lim _{r \rightarrow 1^{-}}\left\langle K_{r, t}(b(T)) x, y\right\rangle \\
= & \lim _{r \rightarrow 1^{-}}\left\langle\left(I d-r e^{-i t} b(T)\right)^{-1} x, y\right\rangle \\
\quad & \quad\left\langle x,\left(I d-r e^{-i t} b(T)\right)^{-1} y\right\rangle-\langle x, y\rangle \\
= & \lim _{r \rightarrow 1^{-}}\left\langle\left(1-r e^{-i t} b\right)^{-1}, x^{T} \cdot y\right\rangle \\
& \quad+\left\langle\left(1-r e^{i t} \bar{b}\right)^{-1}, x^{T} \cdot y\right\rangle-\left\langle 1, x^{T} \cdot y\right\rangle,
\end{aligned}
$$

since $\langle f(T) x, y\rangle=\left\langle f, x^{T} \cdot y\right\rangle$ for all $f \in L^{\infty}$. Hence

$$
\begin{aligned}
\left(x^{b(T)} y\right)\left(e^{i t}\right) & =\lim _{r \rightarrow 1^{-}} \int_{0}^{2 \pi}\left(x^{T} \cdot y\right)\left(e^{i \theta}\right) \frac{1-r^{2}}{\left|1-r e^{-i t} b\left(e^{i \theta}\right)\right|^{2}} \frac{d \theta}{2 \pi} \\
& =\lim _{r \rightarrow 1^{-}} \sum_{j=1}^{d} \int_{J_{j}}\left(x^{T} \cdot y\right)\left(e^{i \theta}\right) \frac{1-r^{2}}{\left|1-r e^{-i t} b\left(e^{i \theta}\right)\right|^{2}} \frac{d \theta}{2 \pi}
\end{aligned}
$$




$$
=\lim _{r \rightarrow 1^{-}} \sum_{j=1}^{d} \int_{0}^{2 \pi} \frac{\left(x^{T} \cdot y\right)\left(b^{-1}\left(e^{i \alpha}\right)\right) e^{i \alpha}}{b^{\prime}\left(b^{-1}\left(e^{i \alpha}\right)\right) b^{-1}\left(e^{i \alpha}\right)} \frac{1-r^{2}}{\left|1-r e^{-i t} e^{i \alpha}\right|^{2}} \frac{d \alpha}{2 \pi},
$$

where $e^{i \alpha}=b\left(e^{i \theta}\right)$. Since $\frac{d \theta}{d \alpha} \geq 0$, we have:

$$
\begin{aligned}
\left(x^{b(T)} y\right)\left(e^{i t}\right) & =\lim _{r \rightarrow 1^{-}} \sum_{j=1}^{d} \int_{0}^{2 \pi} \frac{\left(x^{T} \cdot y\right)\left(b^{-1}\left(e^{i \alpha}\right)\right)}{\left|b^{\prime}\left(b^{-1}\left(e^{i \alpha}\right)\right)\right|} \frac{1-r^{2}}{\left|1-r e^{-i t} e^{i \alpha}\right|^{2}} \frac{d \alpha}{2 \pi} \\
& =\sum_{j=1}^{d} \frac{\left(x^{T} \cdot y\right)\left(\xi_{j}\right)}{\left|b^{\prime}\left(\xi_{j}\right)\right|} .
\end{aligned}
$$

The formula (10) is well-defined as shown by the next result [15].

Theorem H. If $b$ is a Blaschke product (finite or not) then

$$
\text { Zero }\left(b^{\prime}\right) \subset \text { closed convex hull of Zero }(b) \cup\{0\} .
$$

Therefore, if $b$ is a finite Blaschke product, $b^{\prime}(\xi) \neq 0$ for all $\xi \in \mathbb{T}$.

Let us recall a result of J. Bourgain, who solved a problem of Douglas and Rudin [8].

Theorem I. Let $f \in L^{1}, f \not \equiv 0$. Then

$$
f \in H^{2}(\mathbb{T}) \overline{H^{2}(\mathbb{T})} \Longleftrightarrow \log |f| \in L^{1} .
$$

Moreover, $L^{1}=H^{2}(\mathbb{T}) \overline{H^{2}(\mathbb{T})}+\mathbb{C}$.

An immediate corollary is the next result.

Corollary 5.2. Let $f_{0} \in L^{\infty}$ with $\frac{1}{f_{0}} \in L^{\infty}$. Then

$$
L^{1}=H^{2}(\mathbb{T}) \overline{H^{2}(\mathbb{T})}+\mathbb{C} f_{0} .
$$

Proof: It is clear that $\log \left|f_{0}\right| \in L^{1}$. Hence, by Theorem I, since $f_{0}$ is also bounded, it follows that $f_{0} \in H^{\infty}(\mathbb{T}) \overline{H^{\infty}(\mathbb{T})}$, that is, we can write $f_{0}=g_{0} \overline{h_{0}}$, where $g_{0}, h_{0} \in H^{\infty}(\mathbb{T})$. Once more, by Theorem I, we see that for all $F \in L^{1}$ there exist $g, h \in H^{2}(\mathbb{T})$ and $c \in \mathbb{C}$ such that $F=g \bar{h}+c$. Since $f \in L^{1}$, we can therefore write $f / f_{0}=g \bar{h}+c$ as above, and so

$$
f=g g_{0} \overline{h h_{0}}+c f_{0} \in H^{2}(\mathbb{T}) \overline{H^{2}(\mathbb{T})}+\mathbb{C} f_{0} .
$$


Definition 5.3. Let $T \in \mathcal{L}(\mathcal{H})$ be an a.c.c. and let $f \in L^{1}$. If there exist $x, y \in \mathcal{H}$ such that $f=x \cdot y$, we say that $T$ factorizes $f$.

Here is a reformulation of Bourgain's result.

Lemma 5.4. Let $S \in \mathcal{L}(\mathcal{H})$ with $\mathcal{H}=H^{2}(\mathbb{T})$ defined by

$$
S f\left(e^{i t}\right)=e^{i t} f\left(e^{i t}\right) .
$$

Then $S$ factorizes $f \in L^{1}$ if and only if $f \equiv 0$ or $\log |f| \in L^{1}$.

Proof: Since $S$ is isometric and completely nonunitary, in particular $S$ is an a.c.c. The only thing to check is that for $g, h \in H^{2}(\mathbb{T})$, we have $g \stackrel{S}{h} h=g \bar{h}$. By Lemma 3.7 we get:

$\widehat{g \cdot h}(n)= \begin{cases}\left\langle S^{* n} g, h\right\rangle=\frac{1}{2 \pi} \int_{0}^{2 \pi} g\left(e^{i t}\right) \overline{h\left(e^{i t}\right)} e^{-i n t} d t=\widehat{g \bar{h}}(n) \quad n \geq 0 \\ \left\langle S^{|n|} g, h\right\rangle=\frac{1}{2 \pi} \int_{0}^{2 \pi} e^{-i n t} g\left(e^{i t}\right) \overline{h\left(e^{i t}\right)} d t=\widehat{g \bar{h}}(n) \quad n \leq 0 .\end{cases}$

The injectivity of the Fourier transform implies that $g \stackrel{S}{\cdot} h=g \bar{h}$.

A reformulation of Corollary 5.2 in terms of factorization of $L^{1}$ functions is the following.

Lemma 5.5. Let $f_{0} \in L^{\infty}$ with $\frac{1}{f_{0}} \in L^{\infty}$. Let $A \in \mathcal{L}(\mathcal{H})$ be an a.c.c. which factorizes $f_{0}$. Then $T:=S \oplus A$ factorizes $L^{1}$.

Proof: $\quad$ Let $f \in L^{1}$. Using Corollary 5.2, there exist $g, h \in H^{2}(\mathbb{T})$ and $\lambda \in \mathbb{C}$ such that $f=g \bar{h}+\lambda f_{0}$. We have already noticed that $g \bar{h}=g^{S} \cdot h$. Our hypothesis implies that there exist $x, y \in \mathcal{H}$ such that $\lambda f_{0}=x^{A} \cdot y$, and hence $f=(g \oplus x)^{T} \cdot(h \oplus y)$. Indeed, if $T_{1}$ and $T_{2}$ are a.c.c. on $\mathcal{H}_{1}$ and $\mathcal{H}_{2}$ respectively, then for $x_{1}, y_{1} \in \mathcal{H}_{1}$ and $x_{2}, y_{2} \in \mathcal{H}_{2}$, it is not difficult to check that:

$$
\left(x_{1} \oplus x_{2}\right) \stackrel{T_{1} \oplus T_{2}}{\cdot}\left(y_{1} \oplus y_{2}\right)=x_{1} \stackrel{T_{1}}{T_{1}} y_{1}+x_{2} \stackrel{T_{2}}{\cdot} y_{2} .
$$

Now, let us give an immediate corollary of the previous lemma.

Corollary 5.6. Let $A \in \mathcal{L}(\mathcal{H})$ be an a.c.c. such that $\left(\sigma_{p}(A) \cap \mathbb{D}\right) \cup$ $\left(\sigma_{p}\left(A^{*}\right) \cap \mathbb{D}\right) \neq \emptyset$. Then $S \oplus A$ factorizes $L^{1}$.

Proof: $\quad$ Let $\lambda=r e^{i t} \in \sigma_{p}(A) \cap \mathbb{D}$. Then there exists $x \in \mathcal{H}$ such that $\|x\|=1,\left\langle A^{n} x, x\right\rangle=\lambda^{n}$ and $\left\langle A^{* n} x, x\right\rangle=\bar{\lambda}^{n}$. So $A$ factorizes $P_{r, t}$ with $P_{r, t} \in L^{\infty}(\mathbb{T})$ and $\frac{1}{P_{r, t}} \in L^{\infty}(\mathbb{T})$. If $\lambda \in \sigma_{p}\left(A^{*}\right) \cap \mathbb{D}$, then $A$ factorizes $P_{r,-t}$. It remains to apply Lemma 5.5.

A combination of Corollary 5.6 and Theorem 5.1 provides the following factorization result. 
Theorem 5.7. For all $f \in L^{1}$, there exist $g, h \in H^{2}(\mathbb{T})$ such that:

$$
f\left(e^{i t}\right)=\frac{(g \bar{h})\left(e^{i t / 2}\right)+(g \bar{h})\left(-e^{i t / 2}\right)}{2} .
$$

Proof: $\quad$ Since $\sigma_{p}\left(S^{*}\right)=\mathbb{D}$, the operator $S \oplus S$, which is unitarily equivalent to $S^{2}$, factorizes $L^{1}$. Consider the finite Blaschke product $b(z)=z^{2}$. We have seen that, for $g, h \in H^{2}(\mathbb{T})$,

$$
\left(g^{S^{2}} \cdot h\right)\left(e^{i t}\right)=\sum_{k=1}^{2} \frac{(g \cdot h)\left(\xi_{k}\right)}{2},
$$

where $\xi_{1}, \xi_{2}$ are the solutions of $z^{2}=e^{i t}$ and $g \stackrel{S}{h} h=g \bar{h}$. Since $S^{2}$ factorizes $L^{1}, \forall f \in L^{1}$, there exist $g, h \in H^{2}(\mathbb{T})$ such that:

$$
f\left(e^{i t}\right)=\frac{(g \bar{h})\left(e^{i t / 2}\right)+(g \bar{h})\left(-e^{i t / 2}\right)}{2} .
$$

Acknowledgements. The author is grateful to M. Mathieu and A. W. Wickstead who organized the Belfast Functional Analysis Day 2002 , where this survey was presented.

\section{REFERENCES}

[1] C. Apostol. Ultraweakly closed operator algebras. J. Operator Theory, 2:4961,1979 .

[2] B. Beauzamy. Un opérateur sans sous-espace invariant: simplification de l'exemple de P. Enflo. Integral Equations Oper. Theory, 8(3):314-384, 1985.

[3] B. Beauzamy. Propriétés spectrales d'un opérateur sans sous-espace invariant. J. Operator Theory, 16:349-353, 1986.

[4] H. Bercovici. Factorization theorems and the structure of operators on Hilbert space. Annals of Math., 128:399-413, 1988.

[5] H. Bercovici, C. Foias, and C. Pearcy. Dilation theory and systems of simultaneous equations in the predual of an operator algebra, I. Michigan Math. J., 30:335-354, 1983.

[6] H. Bercovici, C. Foias, and C. Pearcy. Dual algebras with applications to invariant subspaces and dilation theory. In $C B M S$ Regional conference series in mathematics, 56. A.M.S., Providence, 1985.

[7] H. Bercovici, C. Foias, and C. Pearcy. Two Banach space methods and dual operator algebras. J. Funct. Anal., 78:306-345, 1988.

[8] J. Bourgain. A problem of Douglas and Rudin on factorization. Pacific J. Math., 121(1):47-50, 1986.

[9] L. Brown, A. Shields, and K. Zeller. On absolutely convergent exponential sums. Trans. Amer. Math. Soc., 96:162-183, 1960.

[10] S. Brown. Some invariant subspaces for subnormal operators. Integral Equations and Operator Theory, 1:310-333, 1978.

[11] S. Brown and B. Chevreau. Toute contraction à calcul fonctionnel isométrique est réflexive. C.R. Acad. Sci. Paris Sér. I Math., 307:185-188, 1988. 
[12] S. Brown, B. Chevreau, and C. Pearcy. On the structure of contraction operators. II. J. Funct. Anal., 76:30-55, 1988.

[13] G. Cassier. Ensembles K-spectraux et algèbres duales d'opérateurs. preprint, LAFP, (2), 1991.

[14] G. Cassier. Champs d'algèbres duales et algèbres duales uniformes d'opérateurs sur l'espace de Hilbert. Studia Math., 106:101-119, 1993.

[15] G. Cassier and I. Chalendar. The group of the invariants of a finite Blaschke product. Complex Variables, 42:193-206, 2000.

[16] G. Cassier, I. Chalendar, and B. Chevreau. New examples of contractions illustrating membership and non-membership in the classes $\mathbb{A}_{n, m}$. Acta Sci. Math. (Szeged), 64:707-731, 1998.

[17] G. Cassier, I. Chalendar, and B. Chevreau. Some mapping theorems for the classes $\mathbb{A}_{n, m}$ and boundary sets. Proc. London Math. Soc., 79(1):222-240, 1999.

[18] G. Cassier and T. Fack. Contractions in Von Neumann Algebras. J. Funct. Anal., 135(2):297-338, 1996.

[19] I. Chalendar and J. Esterle. Le problème du sous-espace invariant. In Development of mathematics 1950-2000, pages 235-267. Birkhäuser, Basel, 2000.

[20] I. Chalendar and F. Jaeck. On the contractions in the classes $\mathbb{A}_{n, m} . J . O p$ erator Theory, 38(2):265-296, 1997.

[21] I. Chalendar and J. R. Partington. Spectral density for multiplication operators with applications to factorization of $L^{1}$ functions. J. Operator Theory. to appear.

[22] I. Chalendar and J. R. Partington. $L^{1}$ factorizations for some perturbations of the unilateral shift. C.R. Acad. Sci. Paris Sér. I Math., 332:115-119, 2001.

[23] B. Chevreau. Sur les contractions à calcul fonctionnel isométrique II. J. Operator Theory, 20:269-293, 1988.

[24] R. E. Curto and F. H. Vasilescu. Automorphism invariance of the operatorvalued Poisson transform. Acta Sci.(Szeged), 57:65-78, 1993.

[25] P. L. Duren. Theory of $H^{p}$ spaces. Academic Press, New York, 1970. Pure and applied mathematics, vol. 38 .

[26] P. Enflo. On the invariant subspace problem in Banach spaces. In Espaces $L^{p}$, applications radonifiantes et géométrie des espaces de Banach, Séminaire Maurey-Schwartz (1975 - 1976). 1976.

[27] P. Enflo. On the invariant subspace problem in Banach spaces. Acta Math., 158:213-313, 1987.

[28] G. Exner and Il Bong Jung. Dual operator algebras and contractions with finite defect indices. J. Operator Theory, 36(1):107-119, 1996.

[29] C. Foiaş. Sur certains théorèmes de J. von Neumann concernant les ensembles spectraux. Acta Sci. Math. Szeged, 18:15-20, 1957.

[30] E. Heinz. Ein v. Neumannscher Satz über beschränkte Operatoren im Hilbertschen Raum. Nachr. Akad. Wiss. Göttingen. Math.-Phys. Kl. IIa. Math.-Phys.-Chem. Abt., pages 5-6, 1952.

[31] K. Hoffman. Banach spaces of analytic functions. Prentice-Hall, Englewood Cliffs, 1965. 
[32] Il Bong Jung. Dual operator algebras and the classes $\mathbb{A}_{m, n}$, I. J. Operator Theory, 27:309-323, 1992.

[33] V. I. Lomonosov. Invariant subspaces for the family of operators which commute with a completely continuous operator. Funct. Anal. Appl., 7:213$214,1973$.

[34] V. I. Lomonosov. An extension of Burnside's theorem to infinite-dimensional spaces. Israel Math. J., 75:329-339, 1991.

[35] V. I. Paulsen. Completely bounded maps and dilations, volume 146 of Pitman Research Notes in Mathematics Series. Longman Scientific \& Technical, Harlow, 1986.

[36] H. Radjavi and P. Rosenthal. Invariant Subspaces. Springer-Verlag Berlin Heidelberg, New-York, 1973.

[37] C. Read. A solution to the invariant subspace problem. Bull. London Math. Soc., 16:337-401, 1984.

[38] C. Read. A solution to the invariant subspace problem on the space $\ell^{1}$. Bull. London Math. Soc., 17:305-317, 1985.

[39] C. Read. A short proof concerning the invariant subspace problem. J. London Math. Soc., 34(2):335-348, 1986.

[40] C. Read. The invariant subspace problem for a class of Banach spaces. II. Hypercyclic operators. Israel J. Math., 63(1):1-40, 1988.

[41] C. Read. The invariant subspace problem on some Banach spaces with separable dual. Proc. London Math. Soc., 58(3):583-607, 1989.

[42] C. Read. Quasinilpotent operators and the invariant subspace problem. J. London Math. Soc., 56(3):595-606, 1997.

[43] F. Riesz and B. Sz.-Nagy. Functional analysis. Frederick Ungar Publishing Co., New York, 1955. Translated by Leo F. Boron.

[44] W. Rudin. Real and complex analysis. McGraw-Hill, third edition, 1986.

[45] B. Sz.-Nagy and C. Foiaş. Sur les contractions de l'espace de Hilbert. IV. Acta Sci. Math. Szeged, 21:251-259, 1960.

[46] B. Sz.-Nagy and C. Foias. Harmonic analysis of operators on Hilbert space. North-Holland, 1970.

[47] F. H. Vasilescu. An operator-valued Poisson kernel. J. Funct.Anal., 110(1):47-72, 1992.

[48] F. H. Vasilescu. Operator-valued Poisson kernels and standard models in several variables. Algebraic methods in operator theory, pages 37-46, 1994.

Isabelle Chalendar,

Institut Girard Desargues,

UFR de Mathématiques,

Université Claude Bernard Lyon 1,

F-69622 Villeurbanne Cedex, France

chalenda@igd.univ-lyon1.fr

Received on 22 January 2003. 